\title{
Local Boundedness of Monotone Bifunctions
}

\author{
Mohammad Hossein Alizadeh ${ }^{1}$ and Nicolas Hadjisavvas ${ }^{2}$
}

\begin{abstract}
We consider bifunctions $F: C \times C \rightarrow \mathbb{R}$ where $C$ is an arbitrary subset of a Banach space. We show that under weak assumptions, monotone bifunctions are locally bounded in the interior of their domain. As an immediate corollary, we obtain the corresponding property for monotone operators. Also, we show that in contrast to maximal monotone operators, monotone bifunctions (maximal or not maximal) can also be locally bounded at the boundary of their domain; in fact, this is always the case whenever $C$ is a locally polyhedral subset of $\mathbb{R}^{n}$ and $F(x, \cdot)$ is quasiconvex and lower semicontinuous.
\end{abstract}

Keywords: Monotone bifunction; equilibrium problem; locally bounded operator

Mathematics Subject Classification 2010: 47H05; 49J53; $47 \mathrm{H} 04$.

\footnotetext{
${ }^{1}$ Department of Product and Systems Design Engineering, University of the Aegean, 84100 Hermoupolis, Syros, Greece. e-mail: alizadeh@aegean.gr

${ }^{2}$ Corresponding author. Department of Product and Systems Design Engineering, University of the Aegean, 84100 Hermoupolis, Syros, Greece. e-mail: nhad@aegean.gr
} 


\section{Introduction}

Let $X$ be a Banach space and $C$ a nonempty subset of $X$. A bifunction $F$ : $C \times C \rightarrow \mathbb{R}$ is called monotone if

$$
\forall x, y \in C, \quad F(x, y)+F(y, x) \leq 0 .
$$

Obviously, a monotone bifunction satisfies $F(x, x) \leq 0$ for all $x \in C$. In most papers on monotone bifunctions one always assumes that

$$
F(x, x)=0, \quad \forall x \in C .
$$

In view of the results to follow, we will only assume that (2) holds in specific cases.

Monotone bifunctions were mainly studied in conjunction with the so-called equilibrium problem: find $x_{0} \in C$ such that

$$
\forall y \in C, \quad F\left(x_{0}, y\right) \geq 0 .
$$

Equilibrium problems were studied in many papers (see $[1,2,4,7,9,10$, $11,12,14]$ and the references therein) after Blum and Oettli showed in their highly influencing paper [5] that equilibrium problems include variational inequalities, fixed point problems, saddle point problems etc. In some of these papers $[2,3,14]$ monotone bifunctions were related to monotone operators (see the next section for details) and maximal monotonicity of bifunctions was defined and studied. In [8] some results on maximal monotonicity of bifunctions were deduced assuming that the bifunction is locally bounded, i.e. its values are bounded from above for all $x, y$ in a suitable neighborhood of each point of $C$ or int $C$.

The aim of this paper is to study local boundedness of monotone bifunctions in relation with the corresponding property of monotone operators. After some preliminary definitions and properties presented in Section 2, we will show in Section 3 that under very weak assumptions, local boundedness of monotone bifunctions is automatic at every point of $\operatorname{int} C$. In this way one can obtain an easy proof of the corresponding property of monotone operators. In addition, we will show in Section 4 that monotone bifunctions are in some ways better behaved that the underlying monotone operators, since they can be locally bounded even at the boundary of their domain of definition. In contrast to this, it is known that maximal monotone operators $T$ whose domain $D(T)$ has nonempty interior are never locally bounded at the boundary of $D(T)$. In fact, we will show that in $\mathbb{R}^{n}$ and for locally polyhedral domains $C$, an automatic local boundedness of bifunctions holds. We also show that each monotone operator is "inward locally bounded" at every point of the closure of its domain, a property which collapses to ordinary local boundedness at interior points of the domain.

In what follows, given a set $C \subseteq X$ we will denote its interior and its closure by $\operatorname{int} C$ and $\bar{C}$, respectively. Also, we denote by $N_{C}(x)$ the normal cone at $x \in C$ :

$$
N_{C}(x)=\left\{x^{*} \in X^{*}:\left\langle x^{*}, y-x\right\rangle \leq 0, \quad \forall y \in C\right\} .
$$


A bifunction $F: C \times C \rightarrow \mathbb{R}$ is called cyclically monotone [8] if

$$
\forall x_{1}, x_{2}, \ldots, x_{n} \in C, \quad F\left(x_{1}, x_{2}\right)+F\left(x_{2}, x_{3}\right)+\ldots+F\left(x_{n}, x_{n+1}\right) \leq 0
$$

where $x_{n+1}:=x_{1}$.

Given a multivalued operator $T: X \rightarrow 2^{X^{*}}$, we will denote by $D(T)$ its domain and by gr $T$ its graph. For two multivalued operators $T$ and $S$ we write $T \subseteq S$ if $S$ is an extension of $T$.

\section{Monotone bifunctions and monotone opera- tors}

Given any bifunction $F: C \times C \rightarrow \mathbb{R}$, one defines the operator $A^{F}: X \rightarrow 2^{X^{*}}$ by

$$
A^{F}(x)=\left\{\begin{array}{cc}
\left\{x^{*} \in X^{*}: \forall y \in C, F(x, y) \geq\left\langle x^{*}, y-x\right\rangle\right\} & \text { if } x \in C, \\
\emptyset & \text { if } x \notin C
\end{array}\right.
$$

(cf. [2]). It is easy to see that if $F$ is monotone, then $A^{F}$ is a monotone operator. Note that whenever $x \in D\left(A^{F}\right),(3)$ and (1) imply that $F(x, x)=0$.

A monotone bifunction $F$ will be called maximal monotone if $A^{F}$ is maximal monotone [8].

We recall that a monotone bifunction $F: C \times C \rightarrow \mathbb{R}$ is called BO-maximal monotone [5], if for every $\left(x, x^{*}\right) \in C \times X^{*}$ the following implication holds:

$$
F(y, x)+\left\langle x^{*}, y-x\right\rangle \leq 0, \quad \forall y \in C \Longrightarrow\left\langle x^{*}, y-x\right\rangle \leq F(x, y), \quad \forall y \in C .
$$

Proposition 1 If $F: C \times C \rightarrow \mathbb{R}$ is maximal monotone, then it is BO-maximal monotone.

Proof. Assume that

$$
F(y, x)+\left\langle x^{*}, y-x\right\rangle \leq 0, \quad \forall y \in C .
$$

Then for every $y \in C$ and $y^{*} \in A^{F}(y)$,

$$
\left\langle x^{*}, x-y\right\rangle \geq F(y, x) \geq\left\langle y^{*}, x-y\right\rangle .
$$

Thus, $\left\langle x^{*}-y^{*}, x-y\right\rangle \geq 0$ holds for each $\left(y, y^{*}\right) \in \operatorname{gr} A^{F}$. Since $A^{F}$ is maximal monotone, $x^{*} \in A^{F}(x)$. Consequently,

$$
F(x, y) \geq\left\langle x^{*}, y-x\right\rangle, \quad \forall y \in C .
$$

Hence, implication (4) holds.

The converse is not true in general [8], but it is true if $C$ is convex, $F(x, \cdot)$ is lower semicontinuous (lsc) and convex for all $x \in C$, and property (2) holds [2]. 
Given an operator $T: X \rightarrow 2^{X^{*}}$, one can define the bifunction $G_{T}: D(T) \times$ $D(T) \rightarrow \mathbb{R}$ by

$$
G_{T}(x, y)=\sup _{x^{*} \in T(x)}\left\langle x^{*}, y-x\right\rangle .
$$

Then $G_{T}$ satisfies (2), and is monotone whenever $T$ is monotone [8].

Thus, to each monotone operator $T$ corresponds a monotone bifunction $G_{T}$, and to each monotone bifunction $F$ corresponds a monotone operator $A^{F}$. It is obvious that $T \subseteq A^{G_{T}}$ for each monotone operator $T$. In general equality does not hold; however if $T$ is maximal monotone, then $T=A^{G_{T}}$ so $G_{T}$ is maximal monotone. More generally, one has:

Proposition 2 Let $T: X \rightarrow 2^{X^{*}}$ be monotone with closed convex values, and such that $\overline{D(T)}$ is convex. For any $x \in D(T)$, set $K(x)=N_{D(T)}(x)$. If $T(x)+$ $K(x) \subseteq T(x)$ for all $x \in D(T)$, then $A^{G_{T}}=T$.

Proof. We have only to prove that $A^{G_{T}}(x) \subseteq T(x)$ for all $x \in X$. Let $x \in X$ and $z^{*} \in A^{G_{T}}(x)$. Then

$$
\sup _{x^{*} \in T(x)}\left\langle x^{*}, y-x\right\rangle \geq\left\langle z^{*}, y-x\right\rangle, \quad \forall y \in D(T) .
$$

Assume that $z^{*} \notin T(x)$. Since $T(x)$ is closed and convex, there exists $v \in X$ such that

$$
\sup _{x^{*} \in T(x)}\left\langle x^{*}, v\right\rangle<\left\langle z^{*}, v\right\rangle
$$

For every $y^{*} \in K(x)$ and every $x^{*} \in T(x)$ one has by assumption $x^{*}+t y^{*} \in$ $T(x)$ for all $t \geq 0$. Hence (7) implies

$$
\forall t \geq 0, \quad\left\langle x^{*}, v\right\rangle+t\left\langle y^{*}, v\right\rangle<\left\langle z^{*}, v\right\rangle .
$$

It follows that $\left\langle y^{*}, v\right\rangle \leq 0$. Therefore $v$ is in the polar cone of $K(x)$, which is equal to the tangent cone $T_{D(T)}(x)$ of $D(T)$ at $x$. Hence $v$ can be written as a limit

$$
v=\lim _{n \rightarrow \infty} \frac{y_{n}-x}{\lambda_{n}}
$$

where $y_{n} \in D(T)$ and $\lambda_{n} \searrow 0$. It also follows from (8) that $\left\langle x^{*}, v\right\rangle<\left\langle z^{*}, v\right\rangle$. Thus for $n$ sufficiently large,

$$
\left\langle x^{*}, y_{n}-x\right\rangle<\left\langle z^{*}, y_{n}-x\right\rangle \text {. }
$$

But this contradicts $(6)$. Hence $z^{*} \in T(x)$.

We remark that whenever $T$ is maximal, its values are closed and convex and $T(x)+K(x) \subseteq T(x)$ for all $x \in D(T)$. If in addition $X$ is reflexive, then $\overline{D(T)}$ is convex so all assumptions of Proposition 2 hold. Another case where the assumptions obviously hold is provided by the following:

Corollary 3 Let $T: X \rightarrow 2^{X^{*}}$ be monotone with closed, convex values and such that $D(T)=X$. Then $A^{G_{T}}=T$. 
Given a monotone operator $T$, one may define another monotone bifunction $\hat{G}_{T}$ by the following procedure which is taken from [13] and is reproduced here for the convenience of the reader. First, define $G_{T}: D(T) \times \operatorname{co} D(T) \rightarrow \mathbb{R} \cup\{\infty\}$ as usual:

$$
G_{T}(x, y)=\sup _{x^{*} \in T(x)}\left\langle x^{*}, y-x\right\rangle, \quad x \in D(T), y \in \operatorname{co} D(T) .
$$

Then define $\hat{G}_{T}: \operatorname{co} D(T) \times \operatorname{co} D(T) \rightarrow \mathbb{R} \cup\{\infty\}$ as the concave hull of $G_{T}(\cdot, y)$ for each $y \in \operatorname{co} D(T)$, i.e.,

$$
\hat{G}_{T}(x, y)=\sup \left\{\sum_{i=1}^{k} \alpha_{i} G_{T}\left(x_{i}, y\right): x=\sum_{i=1}^{k} \alpha_{i} x_{i}, x_{i} \in D(T), \sum_{i=1}^{k} \alpha_{i}=1, \alpha_{i} \geq 0\right\} .
$$

Note that $\hat{G}_{T}$ is well-defined, its values cannot be $-\infty$, and $\hat{G}_{T}(x, \cdot)$ is lsc and convex as supremum of lsc and convex functions.

Proposition $4 \hat{G}_{T}$ is real-valued, monotone, and such that $G_{T}(x, y) \leq \hat{G}_{T}(x, y)$ for all $(x, y) \in D(T) \times \operatorname{co} D(T)$.

Proof. The inequality $G_{T}(x, y) \leq \hat{G}_{T}(x, y)$ for $(x, y) \in D(T) \times \operatorname{co} D(T)$ is obvious from the definition of $\hat{G}_{T}$. Since for $(x, y) \in D(T) \times D(T)$ one has $G_{T}(x, y) \leq-G_{T}(y, x)$ and $-G_{T}(y, \cdot)$ is concave, it follows that

$$
\forall(x, y) \in \operatorname{co} D(T) \times D(T), \quad \hat{G}_{T}(x, y) \leq-G_{T}(y, x) .
$$

Now take the convex hull with respect to $y$ of both sides of (11). $\hat{G}_{T}(x, y)$ remains unchanged since $\hat{G}_{T}(x, \cdot)$ is convex, and $-G_{T}(y, x)$ becomes $-\hat{G}_{T}(y, x)$. It follows that

$$
\hat{G}_{T}(y, x)+\hat{G}_{T}(x, y) \leq 0, \forall(x, y) \in \operatorname{co} D(T) \times \operatorname{co} D(T) .
$$

Thus, $\hat{G}_{T}$ is monotone. Also, it follows from (12) that $\hat{G}_{T}$ is real-valued since $\hat{G}_{T}$ does not take the value $-\infty$.

Note that $\hat{G}_{T}(x, x) \leq 0$ for all $x \in \operatorname{co} D(T)$, while for $x \in D(T)$ one has $\hat{G}_{T}(x, x)=0$ since $\hat{G}_{T}(x, x) \geq G_{T}(x, x)$. It is not true in general that $\hat{G}_{T}(x, x)=0$ for all $x \in \operatorname{co} D(T)$.

We note in passing the relation of the functions $G_{T}$ and $\hat{G}_{T}$ with the Fitzpatrick function [6] and the Krauss saddle function [13]. Given a monotone operator $T$, the Fitzpatrick function $\mathcal{F}_{T}$ is the closed convex function on $X \times X^{*}$ defined by

$$
\mathcal{F}_{T}\left(x, x^{*}\right)=\sup \left\{\left\langle y^{*}, x\right\rangle+\left\langle x^{*}, y\right\rangle-\left\langle y^{*}, y\right\rangle:\left(y, y^{*}\right) \in \operatorname{gr} T\right\} .
$$

Now extend the bifunctions $G_{T}$ and $\hat{G}_{T}$ as functions from $X \times X$ to $\mathbb{R} \cup$ $\{-\infty, \infty\}$ by using the same formulas (9) and (10), respectively, but with $(x, y) \in X \times X$. Then $G_{T}(x, y)=-\infty$ if and only if $x \notin D(T)$, while $\hat{G}_{T}(x, y)=-\infty$ if and only if $x \notin \operatorname{co} D(T)$. 
A simple calculation shows that for all $\left(x, x^{*}\right) \in X \times X^{*}$,

$$
\begin{aligned}
\mathcal{F}_{T}\left(x, x^{*}\right) & =\sup _{y \in D(T)}\left(\left\langle x^{*}, y\right\rangle+\sup _{y^{*} \in T(y)}\left\langle y^{*}, x-y\right\rangle\right) \\
& =\sup _{y \in D(T)}\left(\left\langle x^{*}, y\right\rangle+G_{T}(y, x)\right)=\left(-G_{T}(\cdot, x)\right)^{*}\left(x^{*}\right)
\end{aligned}
$$

i.e., $\mathcal{F}_{T}$ is the convex conjugate of $-G_{T}$ with respect to the first variable. Thus, for $(x, y) \in X \times X$,

$$
\left(-G_{T}(\cdot, x)\right)^{* *}(y)=\sup \left\{\left\langle x^{*}, y\right\rangle-\mathcal{F}_{T}\left(x, x^{*}\right): x^{*} \in X^{*}\right\} .
$$

Now $\left(-G_{T}(\cdot, x)\right)^{* *}$ is the closure (in the sense of Rockafellar [16]) of the convex hull of $-G_{T}(\cdot, x)$, i.e., the closure of $-\hat{G}(\cdot, x)$. On the other hand, for $(x, y) \in \operatorname{co} D(T) \times \operatorname{co} D(T), \hat{G}_{T}(x, y)=K_{T}(x, y)$ where $K_{T}$ is the function defined by Krauss (Definition 6 in [13]).

From now on, we will consider the restrictions of $G_{T}$ and $\hat{G}_{T}$ to $D(T) \times D(T)$ and co $D(T) \times \operatorname{co} D(T)$, respectively. Thus $G_{T}$ and $\hat{G}_{T}$ will be real-valued and monotone.

\section{Local boundedness of monotone bifunctions}

We reproduce the following definition from [8].

Definition 5 A bifunction $F$ is called locally bounded at $x_{0} \in X$ if there exist $\epsilon>0$ and $k \in \mathbb{R}$ such that $F(x, y) \leq k$ for all $x$ and $y$ in $C \cap B\left(x_{0}, \epsilon\right)$. We call $F$ locally bounded on a set $K \subseteq X$ if it is locally bounded at every $x \in K$.

We recall that an operator $T: X \rightarrow 2^{X^{*}}$ is called locally bounded at $x_{0} \in X$ if there exist $\varepsilon>0$ and $k>0$ such that $\left\|x^{*}\right\| \leq k$ for all $x^{*} \in T(x), x \in B\left(x_{0}, \varepsilon\right)$.

Remark 6 a) If a bifunction (not necessarily monotone) $F: C \times C \rightarrow \mathbb{R}$ is locally bounded at $x_{0} \in \operatorname{int} C$, then $A^{F}$ is locally bounded at $x_{0}$. Indeed, assume that $\varepsilon>0$ and $k \in \mathbb{R}$ are such that $B\left(x_{0}, \varepsilon\right) \subseteq C$ and $F(x, y) \leq k$ for all $x, y \in B\left(x_{0}, \varepsilon\right)$. Then for every $x \in B\left(x_{0}, \frac{\varepsilon}{2}\right), x^{*} \in A^{F}(x)$ and $v \in B(0,1)$, one has $x+\frac{\varepsilon}{2} v \in B\left(x_{0}, \varepsilon\right)$ and

$$
k \geq F\left(x, x+\frac{\varepsilon}{2} v\right) \geq \frac{\varepsilon}{2}\left\langle x^{*}, v\right\rangle .
$$

Thus $\left\|x^{*}\right\| \leq \frac{2 k}{\varepsilon}$ and $A^{F}$ is locally bounded at $x_{0}$. The converse is not true in general (see Example 20 at the end of this section and the subsequent discussion).

b) Likewise, given an operator $T$, if $G_{T}$ is locally bounded at $x_{0} \in \operatorname{int} D(T)$, then $T$ is locally bounded at $x_{0}$. Indeed, $A^{G_{T}}$ is locally bounded at $x_{0}$ by the above argument, so $T$ is also locally bounded since $T \subseteq A^{G_{T}}$.

Local boundedness of bifunctions is a useful property. We reproduce here two of the results in [8]. 
Proposition 7 Assume that $X$ is reflexive, $C$ is convex, and $F$ is maximal monotone, locally bounded on $\bar{C}$, and such that $F(x, x)=0$ for all $x \in C$. Then $C \subseteq \overline{D\left(A^{F}\right)}$.

Proposition 8 Let $F$ be maximal monotone, locally bounded on $\operatorname{int} C$ and such that $F(x, x)=0$ for all $x \in C$. If $C \subseteq \overline{D\left(A^{F}\right)}$, then $\operatorname{int} C=\operatorname{int} D\left(A^{F}\right)$.

Note that in [8] all results are stated for reflexive spaces, but in fact the proof of Proposition 8 does not use reflexivity.

We will show that, under mild assumptions, any monotone bifunction is locally bounded in the interior of its domain. We will need the following lemma, which generalizes to quasiconvex functions a well-known property of convex functions.

Lemma 9 Let $X$ be a Banach space and $f: X \rightarrow \mathbb{R} \cup\{\infty\}$ be lsc and quasiconvex. If $x_{0} \in \operatorname{int} \operatorname{dom}(f)$, then $f$ is bounded from above in a neighborhood of $x_{0}$.

Proof. Let $\varepsilon>0$ be such that $\bar{B}\left(x_{0}, \varepsilon\right) \subseteq \operatorname{dom}(f)$. Set $S_{n}=\left\{x \in \bar{B}\left(x_{0}, \varepsilon\right)\right.$ : $f(x) \leq n\}$. Then $S_{n}$ are convex and closed and $\bigcup_{n \in \mathbb{N}} S_{n}=\bar{B}\left(x_{0}, \varepsilon\right)$. By Baire's theorem, there exists $n \in \mathbb{N}$ such that $\operatorname{int} S_{n} \neq \emptyset$. Take any $x_{1} \in \operatorname{int} S_{n}$ and any $x_{2} \neq x_{0}$ such that $x_{2} \in \bar{B}\left(x_{0}, \varepsilon\right)$ and $x_{0} \in \operatorname{co}\left\{x_{1}, x_{2}\right\}$. Choose $n_{1}>$ $\max \left\{n, f\left(x_{2}\right)\right\}$. Then $x_{1} \in \operatorname{int} S_{n_{1}}, x_{2} \in S_{n_{1}}$ hence $x_{0} \in \operatorname{int} S_{n_{1}}$ so $f$ is bounded by $n_{1}$ at a neighborhood of $x_{0}$.

Note that, if in the above lemma $f$ is lsc and convex, then the result is obvious since $f$ is continuous at every interior point of $\operatorname{dom}(f)$.

Theorem 10 Let $X$ be a Banach space, $C \subseteq X$ a set, and $F: C \times C \rightarrow \mathbb{R} a$ monotone bifunction such that for every $x \in C, F(x, \cdot)$ is lsc and quasiconvex. Assume that for some $x_{0} \in \operatorname{int} C$ there exists a neighborhood $B\left(x_{0}, \varepsilon\right) \subseteq C$ such that for each $x \in B\left(x_{0}, \varepsilon\right), F(x, \cdot)$ is bounded from below ${ }^{3}$ on $B\left(x_{0}, \varepsilon\right)$. Then $F$ is locally bounded at $x_{0}$.

Proof. Let $\varepsilon>0$ be as in the assumption and define $g: B\left(x_{0}, \varepsilon\right) \rightarrow \mathbb{R} \cup\{\infty\}$ by

$$
g(y)=\sup \left\{F(x, y): x \in B\left(x_{0}, \varepsilon\right)\right\} .
$$

We show that $g$ is real-valued. Given $y \in B\left(x_{0}, \varepsilon\right)$, for each $x \in B\left(x_{0}, \varepsilon\right)$,

$$
F(x, y) \leq-F(y, x) .
$$

By assumption, there exists $M_{y}$ such that $F(y, x) \geq M_{y}$ for all $x \in B\left(x_{0}, \varepsilon\right)$. Hence $g(y) \leq-M_{y}<\infty$, i.e., $g$ is real-valued.

Now $g$ is lsc and quasiconvex, and $x_{0} \in \operatorname{int} \operatorname{dom}(g)$. By Lemma 9 , we can find $\varepsilon_{1}<\varepsilon$ and $M \in \mathbb{R}$ such that $g(y) \leq M$ for all $y \in B\left(x_{0}, \varepsilon_{1}\right)$. Then $F(x, y) \leq M$ for all $x, y \in B\left(x_{0}, \varepsilon_{1}\right)$ so $F$ is locally bounded at $x_{0}$.

\footnotetext{
${ }^{3}$ This bound may depend on $x$.
} 
Corollary 11 Let $X$ be reflexive and $F: C \times C \rightarrow \mathbb{R}$ be monotone and such that for every $x \in C, F(x, \cdot)$ is lsc and quasiconvex. Then $F$ is locally bounded on $\operatorname{int} C$.

Proof. Given $x_{0} \in \operatorname{int} C$ choose $\varepsilon>0$ such that $\bar{B}\left(x_{0}, \varepsilon\right) \subseteq C$. Since $X$ is reflexive, $\bar{B}\left(x_{0}, \varepsilon\right)$ is weakly compact, hence for each $y \in C, F(y, \cdot)$ has a minimum on $\bar{B}\left(x_{0}, \varepsilon\right)$. Consequently, all assumptions of Theorem 10 are satisfied.

When $F(x, \cdot)$ is lsc and convex, reflexivity of $X$ is not necessary:

Corollary 12 Let $F: C \times C \rightarrow \mathbb{R}$ be monotone and such that for every $x \in C$, $F(x, \cdot)$ is lsc and convex. Then $F$ is locally bounded on $\operatorname{int} C$.

Proof. Let $x_{0} \in \operatorname{int} C$. Choose $\varepsilon>0$ be such that $B\left(x_{0}, \varepsilon\right) \subseteq C$. For every $x \in B\left(x_{0}, \varepsilon\right)$, the subdifferential of $\partial F(x, \cdot)$ is nonempty at $x$. For every $x^{*} \in$ $\partial F(x, \cdot)(x)$ and $y \in B\left(x_{0}, \varepsilon\right)$ one has

$$
F(x, y)-F(x, x) \geq\left\langle x^{*}, y-x\right\rangle \geq-\left\|x^{*}\right\|\|x-y\| \geq-2 \varepsilon\left\|x^{*}\right\| .
$$

Thus $F(x, \cdot)$ is bounded from below on $B\left(x_{0}, \varepsilon\right)$. According to the theorem, $F$ is locally bounded at $x_{0}$.

If $T: X \rightarrow 2^{X^{*}}$ is monotone, then $G_{T}$ is monotone while $G_{T}(x, \cdot)$ is lsc and convex. According to the Corollary 12 and Remark 6, we immediately obtain:

Corollary 13 Let $X$ be a Banach space and $T: X \rightarrow 2^{X^{*}}$ be monotone. Then $T$ is locally bounded at every point of int $D(T)$.

We see that the well-known local boundedness of monotone operators can be shown very easily through Corollary 12 on local boundedness of bifunctions. In fact, whenever property (2) holds, one can also easily show the converse, i.e., provide a proof of Corollary 12 assuming that Corollary 13 is known:

Proposition 14 Assume that $F$ is monotone, satisfies (2) and $F(x, \cdot)$ is lsc and convex for each $x \in C$. Then $F$ is locally bounded on $\operatorname{int} C$.

Proof. Under our assumptions, $A^{F}(x)$ is actually the subdifferential $\partial F(x, \cdot)(x)$ of the lsc and convex function $F(x, \cdot)$ at $x$. It is known that this is nonempty for all $x \in \operatorname{int} C$. Hence, the monotone operator $A^{F}$ is locally bounded on $\operatorname{int} C$.

For each $x_{0} \in \operatorname{int} C$ choose $\varepsilon>0$ and $k \in \mathbb{R}$ such that $B\left(x_{0}, \varepsilon\right) \subseteq C$ and $\left\|y^{*}\right\| \leq k$ for every $y^{*} \in A^{F}(y), y \in B\left(x_{0}, \varepsilon\right)$. Then for each $x, y \in B\left(x_{0}, \varepsilon\right)$ and $y^{*} \in A^{F}(y)$,

$$
F(x, y) \leq-F(y, x) \leq-\left\langle y^{*}, x-y\right\rangle \leq\left\|y^{*}\right\|\|x-y\| \leq 2 \varepsilon k .
$$

Thus $F$ is locally bounded on $\operatorname{int} C$.

In fact, with the same proof as in the above proposition, we obtain the slightly more general result, which is a kind of converse of Proposition 8: 
Proposition 15 Assume that $F$ is a monotone bifunction and $\operatorname{int} C=\operatorname{int} D\left(A^{F}\right)$. Then $F$ is locally bounded on $\operatorname{int} C$.

The above proposition induces the following result, which does not assume lower semicontinuity or quasiconvexity.

Proposition 16 Suppose that $\operatorname{int} C \neq \emptyset$ and $F: C \times C \rightarrow \mathbb{R}$ is maximal monotone, cyclically monotone and satisfies $F(x, x)=0$ for all $x \in C$. Then $F$ is locally bounded on $\operatorname{int} C$.

Proof. Since $F$ is maximal monotone and cyclically monotone, by Proposition $5.2(1)$ of $[8]$ we have

$$
\operatorname{int} C=\operatorname{int} D\left(A^{F}\right) \text {. }
$$

Now, Proposition 15 implies that $F$ is locally bounded on $\operatorname{int} C$.

One can also obtain a well-known generalization of Corollary 13 by using bifunctions. We start with a simple remark:

Lemma 17 Suppose that $X$ is a Banach space and $T: X \rightarrow 2^{X^{*}}$ is monotone. Then

(i) $T \subseteq A^{G_{T}} \subseteq A^{\hat{G}_{T}}$;

(ii) $T=A^{G_{T}}=A^{\hat{G}_{T}}$, if $T$ is maximal monotone.

Proof. (i) $T \subseteq A^{G_{T}}$ is obvious. Since $G_{T}(x, y) \leq \hat{G}_{T}(x, y)$ for all $(x, y) \in$ $C \times \operatorname{co} C$, we deduce that $A^{G_{T}} \subseteq A^{\hat{G}_{T}}$.

(ii) Obvious consequence of (i).

Proposition 18 Suppose that $X$ is a Banach space and $T: X \rightarrow 2^{X^{*}}$ is monotone and $\operatorname{int}(\operatorname{co} D(T)) \neq \emptyset$. Then $T$ is locally bounded on $\operatorname{int}(\operatorname{co} D(T))$.

Proof. We know that $\hat{G}_{T}$ is monotone and $\hat{G}_{T}(x, \cdot)$ is lsc and convex for all $x \in \operatorname{co} D(T)$. Thus by Corollary $12, \hat{G}_{T}$ is locally bounded on $\operatorname{int}(\operatorname{co} D(T))$. It follows from Remark 6 that $A^{\hat{G}_{T}}$ is locally bounded on $\operatorname{int}(\operatorname{co} D(T))$. Now Lemma 17 implies that $T$ is locally bounded on $\operatorname{int}(\operatorname{co} D(T))$.

When the assumptions of lower semicontinuity and quasiconvexity do not hold, then local boundedness may fail, as shown by the following examples.

Example 19 Let $x^{*}$ be a noncontinuous linear functional on $X$ and set $F(x, y)=$ $x^{*}(y-x)$. Then $F$ is a monotone bifunction, which is affine but obviously is not locally bounded at any $x \in X$.

Example 20 Define $F$ on $\mathbb{R} \times \mathbb{R}$ by $F(x, y)=\frac{1}{|y|}-\frac{1}{|x|}$ for $x \neq 0$ and $y \neq 0$, and $F(x, 0)=x=-F(0, x), x \in \mathbb{R}$. Then $F$ is monotone, $F(x, \cdot)$ is lsc for every $x \in \mathbb{R}$, but $F$ is not locally bounded at 0 . In addition, this bifunction is a counterexample to the converse of Proposition 1 and of Remark 6: one can readily show that $F$ is BO-maximal monotone and that $D\left(A^{F}\right)=\{0\}$, with $A^{F}(0)=\{-1\}$. It follows that $F$ is not maximal monotone and also $A^{F}$ is locally bounded at $\{0\}$ while $F$ is not. 
In contrast to the previous example, if a monotone operator $T$ is locally bounded at $x_{0} \in X$, then $G_{T}$ is not only locally bounded but also locally bounded by an arbitrarily small positive number at $x_{0}$. Indeed, if $\left\|x^{*}\right\| \leq k$ for all $x \in B\left(x_{0}, \varepsilon\right), x^{*} \in T(x)$, then for all $x, y \in D(T) \cap B\left(x_{0}, \varepsilon\right)$ and $y^{*} \in T(y)$ we find

$$
G_{T}(x, y) \leq-G_{T}(y, x) \leq-\left\langle y^{*}, x-y\right\rangle \leq 2 \varepsilon k .
$$

\section{Local boundedness at arbitrary points}

In Proposition 7 one asks for the bifunction to be maximal monotone and locally bounded on $\bar{C}$. This assumption seems to be in contradiction with the theory of maximal monotone operators. In fact, if $T: X \rightarrow 2^{X^{*}}$ is a maximal monotone operator and int $D(T) \neq \emptyset$, then $T$ is never locally bounded on elements of the boundary of $D(T)$; indeed, we know that $T(x)+K(x) \subseteq T(x)$ where $K(x):=N_{D(T)}(x)$ is a cone which is not equal to $\{0\}$, for $x$ belonging to the boundary of $D(T)$. However, this does not imply that the maximal monotone bifunction $G_{T}$ is also unbounded at $x_{0}$. In fact, in $\mathbb{R}^{n}$ we have a result of local boundedness at arbitrary points and in particular at boundary points, for more general bifunctions.

Let us denote by $\|x\|_{\infty}$ the sup norm of $x=\left(x_{1}, x_{2}, \ldots, x_{n}\right) \in \mathbb{R}^{n}$,

$$
\|x\|_{\infty}=\max \left\{\left|x_{1}\right|,\left|x_{2}\right|, \ldots,\left|x_{n}\right|\right\},
$$

and by $\bar{B}_{\infty}(x, \varepsilon)$ the closed $\varepsilon$-ball around $x$ with respect to $\|\cdot\|_{\infty}$. We call a subset $C$ of $\mathbb{R}^{n}$ locally polyhedral at $x_{0} \in C$ if there exists $\varepsilon>0$ such that $\bar{B}_{\infty}(x, \varepsilon) \cap C$ is a polytope.

In the following proposition we do not assume that $F$ is monotone.

Proposition 21 Let $C \subset \mathbb{R}^{n}$ be locally polyhedral at $x_{0} \in C$ and $F: C \times C$ $\rightarrow \mathbb{R}$ be a bifunction. If $F(x, \cdot)$ is quasiconvex for each $x \in C$, and $F(\cdot, y)$ is upper semicontinuous (usc) for all $y \in C$, then $F$ is locally bounded at $x_{0}$.

Proof. Choose $\varepsilon>0$ such that $\bar{B}_{\infty}\left(x_{0}, \varepsilon\right) \cap C$ is a polytope. Then there exist $x_{1}, x_{2}, \ldots, x_{k}$ such that

$$
\bar{B}_{\infty}\left(x_{0}, \varepsilon\right) \cap C=\operatorname{co}\left\{x_{1}, x_{2}, \ldots, x_{k}\right\} .
$$

Since $F(x, \cdot)$ is quasiconvex, for all $x$ and $y$ in $\bar{B}_{\infty}\left(x_{0}, \varepsilon\right) \cap C$ we have

$$
F(x, y) \leq \max \left\{F\left(x, x_{1}\right), F\left(x, x_{2}\right), \ldots, F\left(x, x_{k}\right)\right\} .
$$

On the other hand $F\left(\cdot, x_{i}\right)$ is usc and $\bar{B}_{\infty}\left(x_{0}, \varepsilon\right) \cap C$ is a compact set, thus $F\left(\cdot, x_{i}\right)$ attains its maximum on $\bar{B}_{\infty}\left(x_{0}, \varepsilon\right) \cap C$; that is, there exists $M_{i}$ such that

$$
F\left(x, x_{i}\right) \leq M_{i} \text { for } i=1,2, \ldots, k \text { and } x \in \bar{B}_{\infty}\left(x_{0}, \varepsilon\right) \cap C .
$$

Set $M=\max \left\{M_{1}, M_{2}, \ldots, M_{k}\right\}$. Then

$$
F(x, y) \leq M \text { for all } x, y \in \bar{B}_{\infty}\left(x_{0}, \varepsilon\right) \cap C .
$$


This means that $F$ is locally bounded at $x_{0}$.

Note that if $x_{0} \in \operatorname{int} C$ then $C$ is locally polyhedral at $x_{0}$. Hence bifunctions satisfying the assumptions of Proposition 21 are in particular locally bounded in the interior of $C$.

Proposition 22 Let $C \subset \mathbb{R}^{n}$ be locally polyhedral at $x_{0}$ and $F: C \times C \rightarrow \mathbb{R}$ be a monotone bifunction. If $F(x,$.$) is quasiconvex and lsc for all x \in C$, then $F$ is locally bounded at $x_{0}$.

Proof. Choose $\varepsilon>0$ such that $\bar{B}_{\infty}\left(x_{0}, \varepsilon\right) \cap C$ is a polytope. Since $F(x, \cdot)$ is quasiconvex, as the proof of the previous proposition there exist $x_{1}, x_{2}, \ldots, x_{k} \in$ $\bar{B}_{\infty}\left(x_{0}, \varepsilon\right) \cap C$ such that for all $x, y \in \bar{B}_{\infty}\left(x_{0}, \varepsilon\right) \cap C$ we have

$$
F(x, y) \leq \max \left\{F\left(x, x_{1}\right), F\left(x, x_{2}\right), \ldots, F\left(x, x_{k}\right)\right\} .
$$

Since $F(x, y)$ is monotone,

$$
F\left(x, x_{i}\right) \leq-F\left(x_{i}, x\right) \text { for } i=1,2, \ldots, k .
$$

For each $i,-F\left(x_{i}, \cdot\right)$ is usc. Therefore, $-F\left(x_{i}, \cdot\right)$ has a maximum $M_{i}$ on $\bar{B}_{\infty}\left(x_{0}, \varepsilon\right) \cap C$. Set $M=\max \left\{M_{1}, M_{2}, \ldots, M_{k}\right\}$. Then (13) and (14) entail

$$
F(x, y) \leq M \text { for all } x, y \in \bar{B}_{\infty}\left(x_{0}, \varepsilon\right) \cap C,
$$

i.e., $F$ is locally bounded at $x_{0}$.

Thus, if $C$ is a polyhedral set and $F$ satisfies the assumptions of Proposition 21 or 22 , then it is locally bounded on $C$, not only on int $C$. However, the following example shows that this property may fail if $C$ is not locally polyhedral.

Example 23 Set $C=\left\{(\alpha, \beta) \in \mathbb{R}^{2}: \alpha \geq \beta^{4}\right\}$. Define the function $f$ on $\mathbb{R}^{2}$ by

$$
f(\alpha, \beta)=\left\{\begin{array}{cc}
\frac{\beta^{2}}{2 \alpha}, \quad \text { if } \alpha \geq \beta^{4}, \alpha>0 \\
0, \quad \text { if } \alpha=\beta=0 \\
\infty, \quad \text { otherwise. }
\end{array}\right.
$$

This function is lsc and convex (it is the restriction to $C$ of the function in $[16$, p. 83]).

Now define the bifunction $F: C \times C \rightarrow \mathbb{R}$ by $F(x, y)=f(y)-f(x), x, y \in C$. This bifunction $F$ has very nice properties: it is cyclically monotone, $F(x, \cdot)$ is convex and lsc, $F(\cdot, y)$ is concave and usc, it is defined on a closed convex set thus it is maximal monotone (see Proposition 3.1 in [8]). Nevertheless, it is not locally bounded at 0 . Indeed, consider the sequences $x_{n}=(0,0)$ and $y_{n}=\left(\frac{1}{n^{4}}, \frac{1}{n}\right)$. Then $F\left(x_{n}, y_{n}\right) \rightarrow \infty$, hence every neighborhood of 0 contains pairs $x, y$ with $F(x, y)$ as large as we wish.

Since monotone bifunctions can be locally bounded at the boundary of their domain, it is interesting to investigate an analogous property for monotone operators. Given a subset $C \subseteq X$, let us denote by inw $C\left(x_{0}\right):=\bigcup_{\lambda>0} \frac{1}{\lambda}\left(\operatorname{int} C-x_{0}\right)$ 
the set of inward directions of $C$ at $x_{0}$. Note that if $v \in \operatorname{inw} C\left(x_{0}\right)$ then $v$ is also an inward direction at all $x$ sufficiently close to $x_{0}$. Indeed, it is sufficient to take $x \in B\left(x_{0}, \varepsilon\right)$ where $\varepsilon>0$ is such that $B\left(x_{0}+\lambda v, \varepsilon\right) \subseteq C$.

Definition 24 An operator $T: X \rightarrow 2^{X^{*}}$ is called inward locally bounded at $x_{0} \in \overline{D(T)}$ if for each $v \in \operatorname{inw} C\left(x_{0}\right)$ there exist $k>0$ and $\varepsilon>0$ such that for all $x \in B\left(x_{0}, \varepsilon\right) \cap C$ and $x^{*} \in T(x)$, one has $\left\langle x^{*}, v\right\rangle \leq k$.

We remark that if $T$ is inward locally bounded at an interior point $x_{0}$ of $D(T)$, then by the uniform boundedness principle it is locally bounded at $x_{0}$, since inw $D(T)\left(x_{0}\right)=X$.

Proposition 25 A monotone operator $T$ is inward locally bounded at every point of $\overline{D(T)}$.

Proof. Let $x_{0} \in \overline{D(T)}$ and $v \in \operatorname{inw} D(T)\left(x_{0}\right)$ be given. Choose $\lambda>0$ such that $x_{0}+\lambda v \in \operatorname{int} D(T)$. Since $T$ is locally bounded at $x_{0}+\lambda v$, there exist $\varepsilon>0$ and $k>0$ such that $B\left(x_{0}+\lambda v, \varepsilon\right) \subseteq D(T)$ and $\left\|y^{*}\right\| \leq k$ for all $y^{*} \in T(y)$, $y \in B\left(x_{0}+\lambda v, \varepsilon\right)$. For every $x \in B\left(x_{0}, \varepsilon\right) \cap D(T)$, one has $x+\lambda v \in B\left(x_{0}+\lambda v, \varepsilon\right)$. Thus for every $x^{*} \in T(x)$ and $y^{*} \in T(x+\lambda v)$,

$$
\left\langle x^{*}, v\right\rangle=\frac{1}{\lambda}\left\langle x^{*}, x+\lambda v-x\right\rangle \leq \frac{1}{\lambda}\left\langle y^{*}, x+\lambda v-x\right\rangle \leq k\|v\| .
$$

Thus $T$ is inward locally bounded at $x_{0}$.

Comparing this last result with Propositions 21 and 22, we should remark that these propositions imply a somewhat stronger local boundedness than inward local boundedness. Indeed, if $T$ is monotone and $D(T)$ is locally polyhedral, then by Proposition 22 the bifunction $G_{T}$ is locally bounded everywhere; thus, $\left\langle x^{*}, y-x\right\rangle$ is bounded from above for all $x^{*} \in T(x)$ where $x, y$ are near a point $x_{0}$ of the boundary, even if $y-x$ is "outward" rather than inward. This is because whenever $y-x$ is outward, its norm is small, so that $\left\langle x^{*}, y-x\right\rangle$ is bounded even if the norm of $x^{*}$ is large.

\section{References}

[1] Allevi E., Gnudi A., Schaible S., Vespucci M. T.: Equilibrium and least element problems for multivalued functions. Journal of Global Optimization 46, 561-569 (2010).

[2] Ait Mansour, M., Chbani Z., Riahi, H.: Recession bifunction and solvability of noncoercive equilibrium problems. Communications in Applied Analysis 7, 369-377 (2003).

[3] Aoyama, K., Kimura, Y., Takahashi, W.: Maximal monotone operators and maximal monotone functions for equilibrium problems. Journal of Convex Analysis 15, 395-4019 (2008). 
[4] Bianchi, M., Pini, R.: A Note on Equilibrium Problems with Properly Quasimonotone Bifunctions. Journal of Global Optimization 20, 67-76 (2001).

[5] Blum, E., Oettli, W.: From optimization and variational inequalities to equilibrium problems. Mathematics Student 63, 123-145 (1994).

[6] Fitzpatrick, S.: Representing monotone operators by convex functions. Workshop and Miniconference on Functional Analysis and Optimization (Canberra, 1988), pp. 59-65. Austral. Nat. Univ., Canberra (1988).

[7] Flores-Bazan, F.: Existence theorems for generalized noncoercive equilibrium problems: The quasiconvex case. SIAM Journal on Optimization 11, 675-690 (2000).

[8] Hadjisavvas, N., Khatibzadeh, H.: Maximal monotonicity of bifunctions. Optimization 59, 147-160 (2010).

[9] Iusem, A., Kassay, G., Sosa, W.: An existence result for equilibrium problems with some surjectivity consequences. Journal of Convex Analysis 16, 807-826 (2009).

[10] Kassay G.: On Equilibrium Problems. In: Optimization and Optimal Control: Theory and Applications, Chinchuluun A., Pardalos P.M., Enkhbat R., Tseveendorj I. (Eds.), pp. 55-83. Springer-Verlag (2010).

[11] Konnov, I.V.: Regularization method for nonmonotone equilibrium problems. Journal of Nonlinear and Convex Analysis 10, 93-101 (2009).

[12] Konnov, I.V., Dyabilkin, D.A.: Nonmonotone equilibrium problems: coercivity conditions and weak regularization. Journal of Global Optimization DOI: $10.1007 / \mathrm{s} 10898-010-9551-7$.

[13] Krauss, E.: A representation of arbitrary maximal monotone operators via subgradients of skew-symmetric saddle functions. Nonlinear Analysis, Theory, Methods and Applications 9, 1381-1399 (1985).

[14] Moudafi, A., Thera, M.: Proximal and dynamical approaches to equilibrium problem. In: Lecture Notes in Economics and Mathematical Systems, vol. 477, pp.187-201. Springer-Verlag, New York (1999).

[15] Rockafellar, R.T.: Local boundedness of nonlinear monotone operators. Michigan Mathematical Journal 16, 397-407 (1969).

[16] Rockafellar, R.T.: Convex Analysis. Princeton University Press, Princeton, NJ (1970). 